\title{
Adipocyte STAT5 deficiency promotes adiposity and impairs lipid mobilisation in mice
}

\author{
Doris Kaltenecker $^{1,2} \cdot$ Kristina M. Mueller ${ }^{1,2} \cdot$ Pia Benedikt $^{3} \cdot$ Ursula Feiler $^{3}$. \\ Madeleine Themanns ${ }^{1,2}$ - Michaela Schlederer ${ }^{1,4}$ - Lukas Kenner ${ }^{1,4,5}$. \\ Martina Schweiger $^{3}$ • Guenter Haemmerle ${ }^{3}$ • Richard Moriggl Re, $^{1,6}$
}

Received: 15 June 2016 / Accepted: 12 October 2016 /Published online: 17 November 2016

(C) The Author(s) 2016. This article is published with open access at Springerlink.com

\begin{abstract}
Aims/hypothesis Dysfunction of lipid metabolism in white adipose tissue can substantially interfere with health and quality of life, for example in obesity and associated metabolic diseases. Therefore, it is important to characterise pathways that regulate lipid handling in adipocytes and determine how they affect metabolic homeostasis. Components of the Janus kinase (JAK)-signal transducer and activator of transcription (STAT) pathway are involved in adipocyte physiology and pathophysiology. However, the exact physiological importance of the STAT family member STAT5 in white adipose tissue is yet to be determined. Here, we aimed to delineate adipocyte STAT5 functions in the context of lipid metabolism in white adipose tissue.

Methods We generated an adipocyte specific knockout of Stat 5 in mice using the Adipoq-Cre recombinase transgene
\end{abstract}

Doris Kaltenecker and Kristina M. Mueller contributed equally to this study.

Electronic supplementary material The online version of this article (doi:10.1007/s00125-016-4152-8) contains peer-reviewed but unedited supplementary material, which is available to authorised users.

Richard Moriggl

richard.moriggl@lbicr.lbg.ac.at

Ludwig Boltzmann Institute for Cancer Research, Vienna, Austria

2 Institute of Animal Breeding and Genetics, University of Veterinary Medicine Vienna, Veterinärplatz 1, 1210 Vienna, Austria

3 Institute of Molecular Biosciences, University of Graz, Graz, Austria

4 Clinical Institute of Pathology, Medical University of Vienna, Vienna, Austria

5 Unit of Pathology of Laboratory Animals, University of Veterinary Medicine Vienna, Vienna, Austria

6 Medical University of Vienna, Vienna, Austria followed by in vivo and in vitro biochemical and molecular studies.

Results Adipocyte-specific deletion of Stat 5 resulted in increased adiposity, while insulin resistance and gluconeogenic capacity was decreased, indicating that glucose metabolism can be improved by interfering with adipose STAT5 function. Basal lipolysis and fasting-induced lipid mobilisation were diminished upon STAT5 deficiency, which coincided with reduced levels of the rate-limiting lipase of triacylglycerol hydrolysis, adipose triglyceride lipase (ATGL, encoded by Pnpla2) and its coactivator comparative gene identification 58 (CGI-58). In a mechanistic analysis, we identified a functional STAT5 response element within the Pnpla2 promoter, indicating that Pnpla2 is transcriptionally regulated by STAT5.

Conclusions/interpretation Our findings reveal an essential role for STAT5 in maintaining lipid homeostasis in white adipose tissue and provide a rationale for future studies into the potential of STAT5 manipulation to improve outcomes in metabolic diseases.

Keywords Adipose tissue · Energy metabolism · Lipolysis · STAT5

$\begin{array}{ll}\text { Abbreviations } \\ \text { ATGL } & \text { Adipose triglyceride lipase } \\ \text { BAT } & \text { Brown adipose tissue } \\ \text { CGI-58 } & \text { Comparative gene identification-58 } \\ \text { ChIP } & \text { Chromatin immunoprecipitation } \\ \text { EWAT } & \text { Epididymal white adipose tissue } \\ \text { FA } & \text { Fatty acid } \\ \text { GH } & \text { Growth hormone } \\ \text { GHR } & \text { Growth hormone receptor } \\ \text { HSL } & \text { Hormone-sensitive lipase }\end{array}$




$\begin{array}{ll}\text { ITT } & \text { Insulin tolerance test } \\ \text { JAK } & \text { Janus kinase } \\ \text { PKA } & \text { Protein kinase A } \\ \text { PTT } & \text { Pyruvate tolerance test } \\ \text { RE } & \text { Response element } \\ \text { ScWAT } & \text { Subcutaneous white adipose tissue } \\ \text { STAT } & \text { Signal transducer and activator of transcription } \\ \text { TG } & \text { Triacylglycerols } \\ \text { WAT } & \text { White adipose tissue }\end{array}$

\section{Introduction}

White adipose tissue (WAT) has crucial functions in maintaining whole body energy homeostasis, and its unique lipid storage capacity helps to prevent ectopic lipid deposition and lipotoxicity [1,2]. At times of energy surplus, NEFAs are deposited as triacylglycerols (TGs) in adipocytes for storage within cellular lipid droplets. Conversely, when energy becomes scarce, TGs are hydrolysed in a tightly controlled process known as lipolysis [2,3]. Disrupting the delicate balance between lipid storage and mobilisation results in dysfunctional WAT and abnormalities in systemic lipid partitioning, which form the basis of metabolic diseases, for example in obesity and lipodystrophy $[1,4]$. Since the storage and release of lipids are critical determinants of WAT integrity and organismal energy homeostasis, these processes are under tight regulation by the central nervous system as well as by counter-regulatory hormones $[1,2]$. While insulin promotes TG storage, catecholamines induce lipolysis through $\beta$-adrenergic activation of protein kinase A (PKA). PKA promotes lipolysis by phosphorylating hormone-sensitive lipase (HSL; encoded by Lipe) and indirect activation of adipose triacylglycerol lipase (ATGL; encoded by Pnpla2) by phosphorylating the lipid droplet coating protein perilipin 1 . Subsequently, perilipin 1 releases comparative gene identification-58 (CGI-58) from its interaction at the lipid droplet surface, thereby enabling CGI-58 to act as ATGL coactivator [5]. Although the hormonal control of lipase activation is well understood, less is known about how lipid mobilisation is transcriptionally controlled in adipocytes.

Transcription factors and associated co-regulators operate in concert to regulate metabolic pathways by modulating target gene expression via integrating endocrine, paracrine and metabolic signals [6-8]. Components of the Janus kinase (JAK)-signal transducer and activator of transcription (STAT) pathway contribute to adipocyte physiology and the pathophysiology of dysfunctional adipose tissue [9-14]. STAT5A and STAT5B (collectively referred to as STAT5) are components of the JAK-STAT pathway that are involved in adipocyte development $[13,15,16]$. However, the functions regulated by STAT5 in mature adipocytes and their consequences in vivo are largely unknown. Adipose STAT5 is prominently activated by growth hormone (GH) via JAK2 [13]. GH induces lipolysis in WAT [17, 18]; circulating levels of GH negatively correlate with WAT mass $[19,20]$ and adipocyte-specific deletion of Jak2 consistently blocks GHinduced lipolysis $[14,21]$. GH deficiency or defective GH receptors (GHRs) result in increased adiposity; in line with these findings, GH levels are frequently reduced in common obesity $[18,19]$. Although these observations implicate STAT5 in WAT lipid metabolism, the functional importance of adipocyte STAT5 has not been genetically defined, nor have its downstream molecular mechanisms been determined.

Here, we investigated the role of STAT5 in WAT lipid homeostasis using an adipocyte-specific gene knockout in mice. To prevent interference with adipogenesis due to STAT5 deficiency, we used the Adipoq-Cre transgenic mice to restrict Stat5 deletion to mature adipocytes [22].

\section{Methods}

Animal experiments Adipocyte-specific STAT5-deficient mice $\left(\right.$ Stat $\left.5^{\text {Adipoq }}\right)$ were generated by crossing Adipoq-Cre [8] to Stat5a/b floxed mice [23]. Mice were housed under standardised conditions ( $12 \mathrm{~h}$ dark/12 h light cycle). Unless stated otherwise, experiments used 2-month-old male Stat ${ }^{\text {Adipoq }}$ mice or Adipoq-Cre negative littermates (controls; C57BL/6 background) fed a standard diet ad libitum. Animal experimentation was approved by the institutional ethics and animal welfare committee and the national authority according to Section 26 of the Animal Experiments Act (Tierversuchsgesetz 2012). Body composition was determined using an EchoMRI-100H system (EchoMRI, Houston, TX, USA). For insulin tolerance tests (ITTs), $0.75 \mathrm{U} / \mathrm{kg}$ insulin was i.p. injected into $4 \mathrm{~h}$ fasted mice. For OGTTs and pyruvate tolerance tests (PTTs), $1 \mathrm{~g} / \mathrm{kg}$ glucose was administered via oral gavage or $2 \mathrm{~g} / \mathrm{kg}$ pyruvate was i.p. injected into overnight fasted mice. The HOMA-IR was calculated from insulin and glucose levels in $4 \mathrm{~h}$ fasted mice: (fasting glucose $[\mathrm{mmol} / \mathrm{l}] \times$ fasting insulin $[\mathrm{pmol} / 1]) \div 135$. Acute $\beta$-adrenergic stimulation was performed by i.p. injection of $1 \mathrm{mg} / \mathrm{kg} \mathrm{CL}-316243$. Blood was collected $20 \mathrm{~min}$ after injection.

Histological analysis Formaldehyde-fixed tissues were dehydrated, paraffin-embedded, sliced, stained with haematoxylin and eosin using standard procedures and analysed by light microscopy. Adipocyte sizes were quantified from at least four different fields per mouse using ImageJ (Bethesda, MD, USA). STAT5 staining was performed as previously described [24].

Metabolite measurements Blood glucose and $\beta$-ketones were measured directly from tail vein blood using a 
glucometer (Abbott, Chicago, IL, USA). All other metabolites were measured with commercial colorimetric assays or ELISA (listed in electronic supplementary material [ESM] Methods, Metabolite measurements).

Quantitative real-time PCR and western blotting For RNA extraction, the RNeasy Lipid Tissue Mini Kit (Qiagen, Venlo, Netherlands) was used for tissue samples and Trizol (Thermo Fisher Scientific, Waltham, MA, USA) for 3T3-L1 adipocyte samples. RNA was reverse transcribed and cDNA was subjected to quantitative real-time PCR (qPCR) using the CFX96 Real-Time System (BioRad, Hercules, CA, USA). Samples were run in duplicate. Primers are listed in ESM Table 1. Protein extraction and western blots (40 $\mu$ g protein samples) were performed as previously described [25]; antibody suppliers are listed in ESM Methods, Western blotting.

Ex vivo measurement of lipolysis Lipolysis of WAT explants was measured as previously described [26]. Explants were stimulated with $10 \mu \mathrm{mol} / 1$ isoprenaline or $500 \mathrm{ng} / \mathrm{ml} \mathrm{GH}$.

Cell culture and adipocyte isolation Differentiated 3T3-L1 adipocytes were incubated with $\mathrm{GH}(500 \mathrm{ng} / \mathrm{ml})$ and/or an equimolar concentration of insulin for $6 \mathrm{~h}$. For luciferase reporter assays, transfections were performed with Lipofectamine 2000 (Thermo Fisher Scientific). Cells were lysed in passive lysis buffer and luciferase activities were measured using the Dual Luciferase Reporter Assay system (Promega, Fitchburg, WI, USA). The generation of reporter constructs is described in ESM Methods, Cell culture and adipocyte isolation. Adipocyte isolation was performed using standard procedures and is described in ESM Methods, Cell culture and adipocyte isolation.

\section{Electrophoretic mobility shift assay and chromatin immu-} noprecipitation Electrophoretic mobility shift assay [25] and chromatin immunoprecipitation (ChIP) [27] were performed as previously described. Detailed description is available in ESM Methods, Electrophoretic mobility shift assay and chromatin immunoprecipitation.

Statistical analysis Results are presented as means \pm SEM. Two-tailed Student's $t$ tests and Wilcoxon rank-sum tests were used for comparing two groups and one-way ANOVA followed by Tukey's honestly significant difference, Dunn's multiple comparison or Bonferroni post hoc tests were used for comparing multiple groups. Tolerance tests were analysed with repeated measures two-way ANOVA followed by Bonferroni post hoc testing. Levels of statistical significance were set at $* p<0.05, * * p<0.01$ and $* * * p<0.001$.

\section{Results}

Adipose STAT5 deficiency results in increased adiposity and reduced basal lipolysis Immunostaining showed STAT5 levels were significantly reduced in epididymal WAT (EWAT), subcutaneous WAT (ScWAT) and interscapular brown adipose tissue (BAT) of Stat $5^{\text {Adipoq }}$ mice, but were unaffected in the liver (ESM Fig. 1a). Western blot analysis confirmed that STAT5 was barely detectable in extracts of isolated white adipocytes and in BAT from Stat $5^{\text {Adipoq }}$ mice (Fig. 1a, ESM Fig. 1b). Eight-week-old male Stat $5^{\text {Adipoq }}$ mice had a greater total fat mass compared with littermate controls, while their lean mass and body weight were unaltered (Fig. 1b). EWAT, BAT, liver and gastrocnemius muscle weight was unchanged, but ScWAT weight was significantly increased (Fig. 1c, ESM Fig. 1c, d). Stat ${ }^{\text {Adipoq }}{ }^{\text {EWAT contained }}$ a higher proportion of large adipocytes (Fig. 1d). Conversely, subcutaneous adipocytes from knockout and control animals were similar in size, suggesting that the higher amount of ScWAT in Stat $5^{\text {Adipoq }}$ mice was due to increased adipocyte numbers. However, levels of the adipocyte differentiation marker proteins peroxisome proliferator-activated receptor $\gamma$ $(\mathrm{PPAR} \gamma)$ and CCAAT/enhancer binding proteins (C/EBP) $\alpha$ and $\delta$ in ScWAT were similar between genotypes (ESM Fig. $1 \mathrm{e}, \mathrm{f}) . \mathrm{C} / \mathrm{EBP} \alpha$ content was increased only in EWAT. In contrast, mRNA levels for genes involved in fatty acid (FA) and TG synthesis, such as acetyl-CoA carboxylase (Acaca), fatty acid synthase (Fasn), PEPCK (Pck1) and diacylglycerol O-acyltransferase 2 (Dgat2), were decreased in both WAT compartments of Stat $5^{\text {Adipoq }}$ mice (Fig. 1e, f). An increase in neither total STAT3 levels nor phosphorylation status was detectable in Stat $5^{\text {Adipoq }}$ WAT, suggesting that a compensatory increase in STAT3 function does not occur in response to adipose STAT5-deficiency (ESM Fig. 1g). In addition, neither typical inflammatory mRNA marker levels in WAT nor plasma IL6 and TNF- $\alpha$ levels were significantly different between genotypes (ESM Fig. 1h-k). Furthermore, macrophage-related transcripts were not differentially expressed in WAT. Although not significant, plasma leptin concentration was slightly increased, while adiponectin concentration was mildly decreased (ESM Fig. 11, m). GH levels were similar between genotypes, as were plasma TG and blood $\beta-$ ketone levels (ESM Fig. 1n-p). Notably, circulating NEFA and glycerol levels were markedly decreased, indicating reduced lipid mobilisation in Stat $5^{\text {Adipoq }}$ mice (Fig. 1g, h). Consistent with this, basal lipolysis (determined by NEFA and glycerol release from WAT explants of fed mice) was reduced upon STAT5 deficiency (Fig. 1i, j). Therefore, our results show that adipose STAT5 deficiency increases body fat mass, which coincides with a decrease in basal lipolysis.

Improved variables of glucose metabolism in Stat $^{\text {Adipoq }}$ mice Given the interdependence of lipid and glucose 
Fig. 1 Adipocyte STAT5 deficiency promotes increased adiposity and decreased basal lipolysis. (a) Western blotting of isolated epididymal and subcutaneous (sc) white adipocyte protein lysates. (b) Body composition analysis $(n \geq 8)$. (c) Fat depot weight in relation to body weight (BW) $(n \geq 7)$. (d) Haematoxylin and eosin staining of WAT. Scale bar, $100 \mu \mathrm{m}$. Cell size was quantified using ImageJ $(n \geq 6)$. (e, f) Relative mRNA content for genes involved in lipid synthesis in (e) EWAT and (f) ScWAT. $\mathrm{C}_{\mathrm{t}}$ values were normalised to Gapdh mRNA levels $(n \geq 5)$. (g) Plasma NEFA and (h) glycerol levels $(n \geq 6)$. (i) Basal lipolysis as indicated by NEFA and (j) glycerol release from WAT explants $(n \geq 6) . * p<0.05$, $* * p<0.01, * * * p<0.001$. White bars, control; black bars, Stat $^{\text {Adipoq }}$. Rel, relative
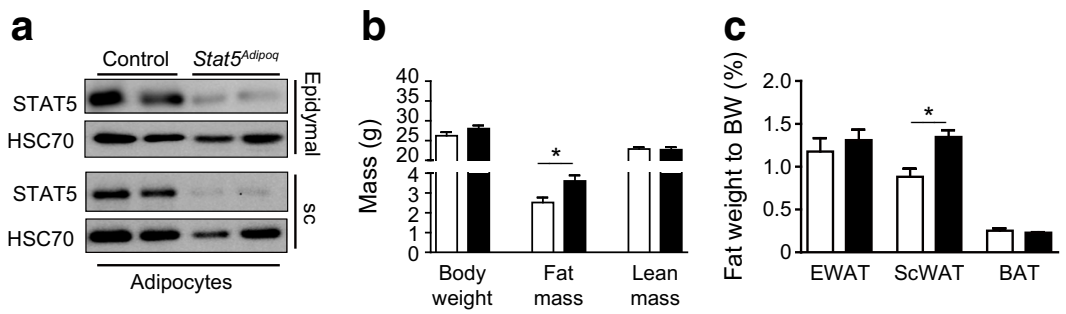

d
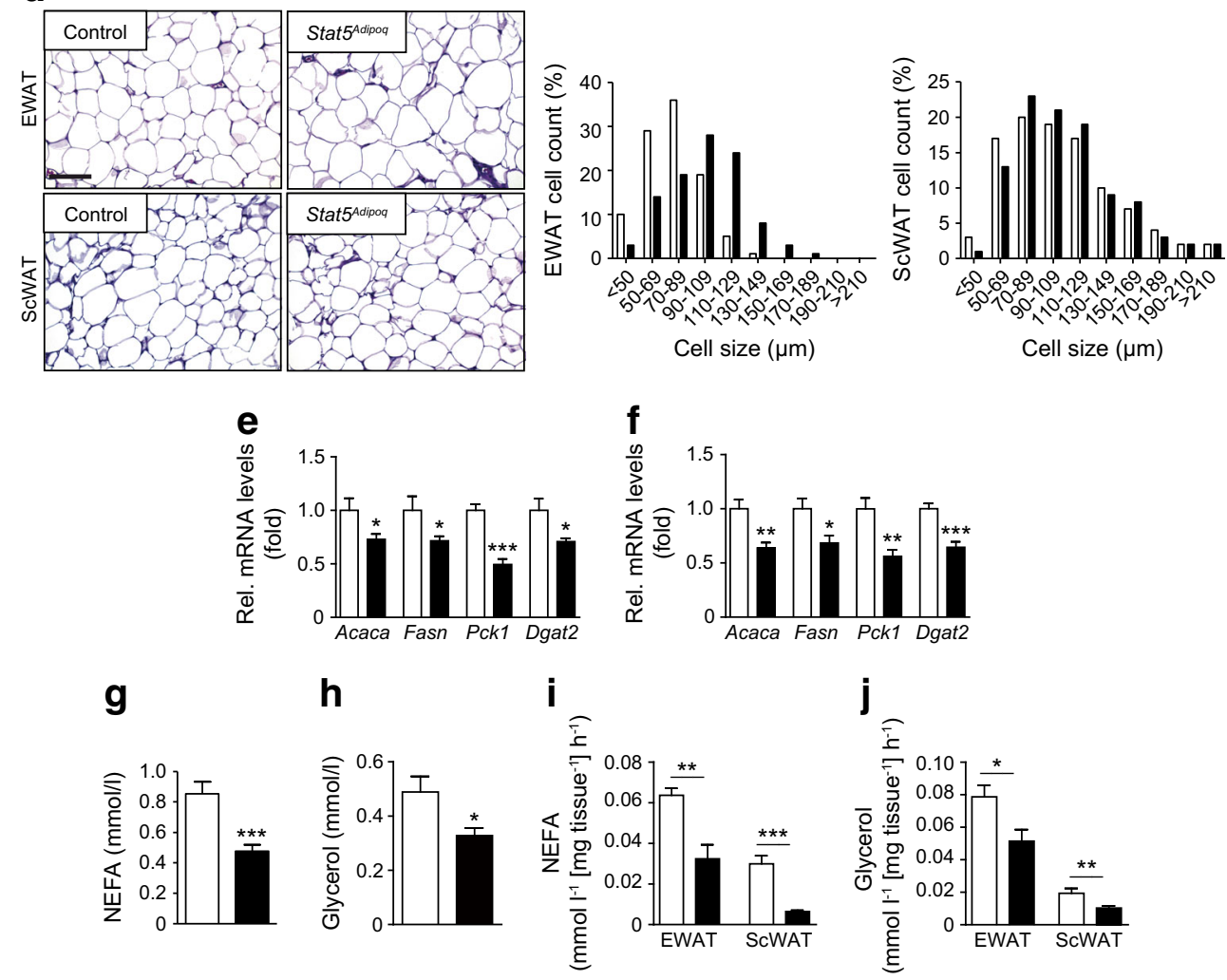

metabolism, we next addressed the impact of adipose STAT5 deficiency on systemic glucose homeostasis. Four h fasted Stat $5^{\text {Adipoq }}$ and control mice had similar blood glucose levels, despite Stat $5^{\text {Adipoq }}$ mice having lower insulin levels, consistent with their lower HOMA-IR (Fig. 2a-c). Liver glycogen stores were reduced in Stat $5^{\text {Adipoq }}$ mice (Fig. 2d), suggesting that either glycogenolysis is increased or glycogen synthesis is reduced to maintain normoglycaemia. OGTTs revealed no difference in glucose clearance between Stat $5^{\text {Adipoq }}$ and control littermates (Fig. 2e). However, post-hypoglycaemic recovery in Stat $5^{\text {Adipoq }}$ mice during ITTs was delayed: glucose levels reached only $60 \%$ of starting values at the end of the experiment (Fig. 2f). As this observation suggests a reduction in counter-regulatory mechanisms such as hepatic glucose production, we performed PTTs to measure hepatic gluconeogenesis indirectly. In agreement with ITT findings, glucose production from pyruvate was reduced in
Stat $5^{\text {Adipoq }}$ mice (Fig. 2g). Interestingly, activating Akt S473 phosphorylation upon insulin injection was similar in liver and WAT lysates from both genotypes (Fig. 2h).

As ageing is linked to deterioration in glucose metabolism, we investigated whether STAT5 deficiency also has beneficial metabolic effects at older ages. Similar to young mice, total fat mass and ScWAT mass were increased in 52-week-old Stat $5^{\text {Adipoq }}$ male mice (Fig. 3a; ESM Fig. 2a). Plasma NEFA and glycerol levels remained lower and blood $\beta$-ketones were reduced in aged Stat $5^{\text {Adipoq }}$ mice (Fig. 3b-d). Although fasting blood glucose levels were decreased in aged Stat $5^{\text {Adipoq }}$ mice, glucose tolerance was similar between genotypes (Fig. 3e, f). However, the increased insulin sensitivity was preserved in aged Stat $5^{\text {Adipoq }}$ animals, as was their reduced capacity to produce glucose from pyruvate (Fig. 3g, h).

Together, these data show that adipose STAT5 deficiency results in decreased insulin resistance scores and reduced gluconeogenic capacity. 
Fig. 2 Variables of glucose metabolism are improved in Stat $5^{\text {Adipoq }}$ mice. (a) Blood glucose and (b) plasma insulin levels in $4 \mathrm{~h}$ fasted mice $(n \geq 6)$. (c) HOMA-IR was calculated from glucose and insulin levels of $4 \mathrm{~h}$ fasted mice $(n \geq 6)$. (d) Liver glycogen content in fed mice $(n \geq 6)$. (e) OGTT: $1 \mathrm{~g} / \mathrm{kg}$ glucose was administered via oral gavage in $16 \mathrm{~h}$ fasted mice. (f) ITT: $0.75 \mathrm{U} / \mathrm{kg}$ insulin was i.p. injected into $4 \mathrm{~h}$ fasted mice. (g) PTT: $2 \mathrm{~g} / \mathrm{kg}$ pyruvate was i.p. injected into $16 \mathrm{~h}$ fasted mice $(n \geq 7)$. (h) Western blotting of insulinstimulated $(0.75 \mathrm{U} / \mathrm{kg}$ body weight) Akt phosphorylation in liver and EWAT lysates. $* p<0.05$; * $p<0.01$. White bars and circles, control; black bars and squares, Stat $5^{\text {Adipoq }}$
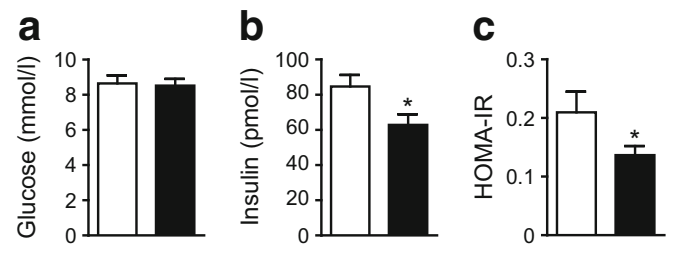

d

e

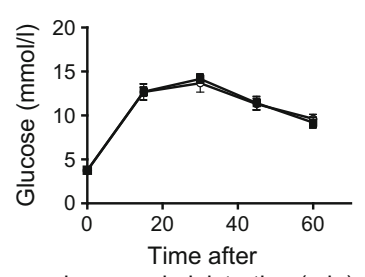

glucose administration ( $\mathrm{min}$ )

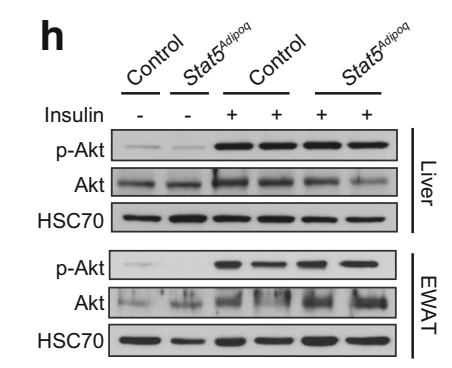

f

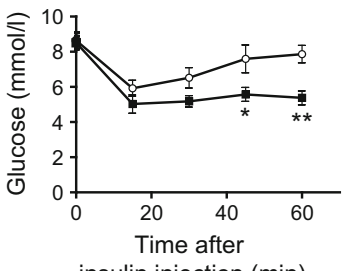

insulin injection (min)
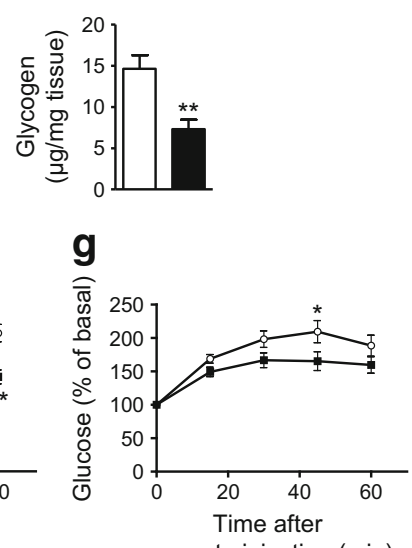

pyruvate injection (min)
Acute $\beta$-adrenergic induction of lipolysis is not impaired in Stat $^{\text {Adipoq }}$ mice Our results demonstrated that basal lipolytic capacity was reduced upon STAT5 deficiency. We next determined the lipolytic response to acute $\beta$-adrenergic stimulation by treating WAT explants with isoprenaline. NEFA and glycerol levels in media from isoprenaline-stimulated Stat $5^{\text {Adipoq }}$ WAT explants were reduced (Fig. 4a, b, ESM Fig. 3a, b), while the fold induction of isoprenaline- stimulated lipolysis over baseline was increased (Fig. 4c, d). Lipolytic products were also reduced upon $\mathrm{GH}$ stimulation of Stat $5^{\text {Adipoq }}$ WAT explants. However, GH-mediated induction of lipolysis was only significantly decreased in STAT5deficient ScWAT explants (Fig. 4a-d). To determine the responses to acute $\beta$-adrenergic stimulation in vivo, we treated mice with CL-316243 (a $\beta_{3}$-adrenergic agonist). Although the fold induction of lipolysis was similar among genotypes,
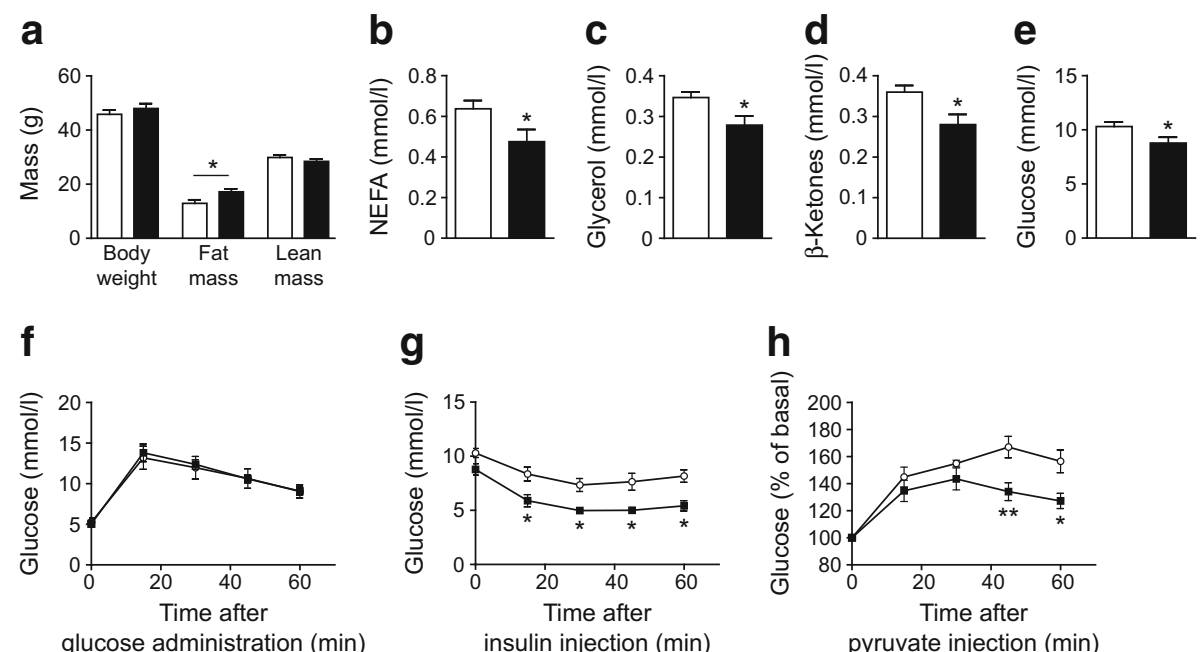

Fig. 3 Improved insulin sensitivity in aged Stat $5^{\text {Adipoq }}$ mice. (a) Body composition analysis $(n=5)$, (b) plasma NEFA and (c) glycerol $(n \geq 7)$, (d) blood $\beta$-ketone and (e) glucose levels in $4 \mathrm{~h}$ fasted 52-week-old mice $(n=10)$. (f) OGTT: $1 \mathrm{~g} / \mathrm{kg}$ glucose was administered via oral gavage in $16 \mathrm{~h}$ fasted mice. (g) ITT: $0.75 \mathrm{U} / \mathrm{kg}$ insulin was i.p. injected into $4 \mathrm{~h}$

fasted mice. (h) PTT: $2 \mathrm{~g} / \mathrm{kg}$ pyruvate was i.p. injected into $16 \mathrm{~h}$ fasted mice $(n \geq 4)$. All tolerance tests were performed in 52 -week-old mice. $* p<0.05, * * p<0.01$. White bars and circles, control; black bars and squares, Stat $5^{\text {Adipoq }}$ 
a

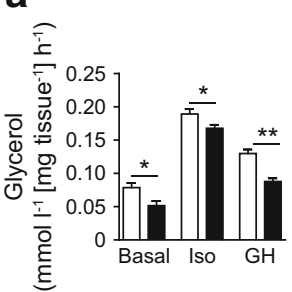

b

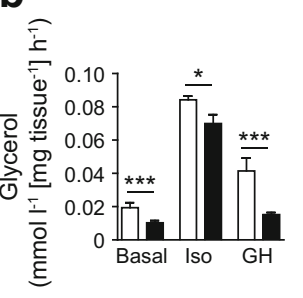

C

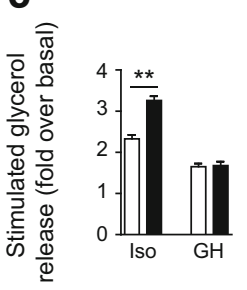

e

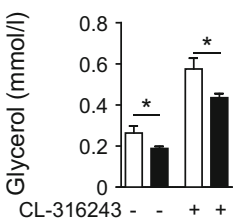

d
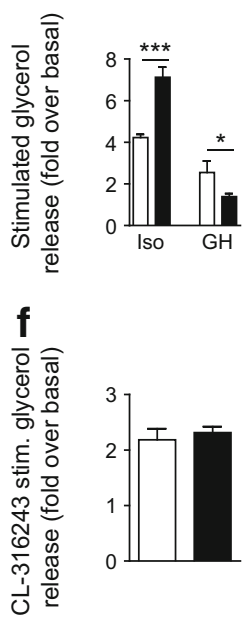

Fig. 4 Acute $\beta$-adrenergic induction of lipolysis is not impaired in Stat $5^{\text {Adipoq }}$ mice. (a, b) Basal and isoprenaline (Iso)- and GH-stimulated lipolysis represented by NEFA and glycerol release from (a) EWAT and (b) ScWAT explants $(n \geq 5)$. (c, d) Fold change in glycerol levels over basal after stimulation of (c) EWAT and (d) ScWAT explants. (e) CL316243 -stimulated glycerol levels in plasma $(n \geq 5)$. (f) Fold change in glycerol levels over basal after CL-316243 treatment $(n \geq 5)$. $* p<0.05$, $* * p<0.01, * * * p<0.001$. White bars, control; black bars, Stat $5^{\text {Adipoq }}$. Stim, stimulated

Stat $5^{\text {Adipoq }}$ mice displayed a $\sim 25 \%$ decrease in plasma glycerol and NEFA levels upon CL-316243 treatment (Fig. 4e, f, ESM Fig. 3c). These findings indicate that $\beta$-adrenergic responsiveness in Stat $5^{\text {Adipoq }}$ mice is intact, although the resulting induction rate is not sufficient to restore circulating lipolytic products to control levels.

Adipocyte-specific STAT5 deficiency impairs fastinginduced lipid mobilisation Given the inability of Stat $5^{\text {Adipoq }}$ mice to compensate for defective basal release of lipolytic products upon acute $\beta$-adrenergic stimulation, we determined their ability to respond to a situation that requires lipolysis by subjecting them to a $48 \mathrm{~h}$ fast. There was no difference in overall body weight loss between fasted Stat $5^{\text {Adipoq }}$ and con-

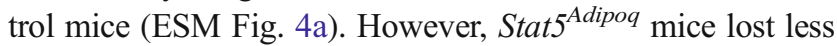
fat mass and more lean mass during this intervention (Fig. 5a, b, ESM Fig. 4b, c). Accordingly, loss of WAT mass was decreased and loss of liver mass was increased in fasted Stat ${ }^{\text {Adipoq }}$ mice, while no changes were observed in gastrocnemius muscle and BAT weight (Fig. 5c-e, ESM Fig. 4d-h). Their inefficient lipid mobilisation was further supported by
WAT histological analysis (Fig. 5f): control adipocytes displayed drastic cell shrinkage upon fasting compared with the fed state, whereas STAT5-deficient adipocytes were almost unaffected by fasting. Plasma NEFA and glycerol concentrations remained lower in $48 \mathrm{~h}$ fasted Stat $5^{\text {Adipoq }}$ mice compared with controls and fasting-associated lipid accumulation was reduced within Stat $5^{\text {Adipoq }}$ livers (Fig. 5g-i, ESM Fig 4i). Plasma TG levels were elevated and GH levels showed a slight but non-significant increase in fasted Stat $5^{\text {Adipoq }}$ mice (Fig. 5j, k). Despite the reduction in lipolytic products as substrates for energy production, fasting blood glucose and $\beta$-ketone levels were similar among genotypes (Fig. 51, m), suggesting that catabolism of non-adipose tissue in Stat $^{\text {Adipoq }}$ mice (i.e. the liver) might compensate for missing substrates that are usually provided by WAT lipid mobilisation. Taken together, our results suggest that adipose STAT5 is required not only for basal lipolysis but also for efficient lipid mobilisation under $48 \mathrm{~h}$ fasting conditions.

Impaired lipolysis in Stat ${ }^{\text {Adipoq }}$ mice is linked to reduced ATGL and CGI-58 levels To gain insight into the molecular changes underlying the lipolytic defect of fed and $48 \mathrm{~h}$ fasted Stat $5^{\text {Adipoq }}$ mice, we measured the mRNA levels for key lipolytic genes in EWAT. No changes were detected in fed or fasting Lipe (encoding HSL), Mgll (encoding monoacylglycerol lipase) and Plin1 (encoding perilipin 1) mRNA levels in STAT5-deficient compared with control EWAT. In contrast, mRNA levels of Pnpla2 (encoding ATGL) and its coactivator Abhd5 (encoding CGI-58) were diminished in the fed state (Fig. 6a-c, ESM Fig. 5a, b). Although fasting significantly increased Pnpla2 and Abhd5 expression in both genotypes, levels in Stat $5^{\text {Adipoq }}$ mice remained significantly lower, indicating that the baseline defect cannot be efficiently compensated for by fasting. Notably, ATGL and CGI-58 protein levels were decreased in EWAT from fed and fasted Stat $5^{\text {Adipoq }}$ mice, whereas phosphorylation status and total levels of HSL as well as PKA activity were similar between the genotypes (Fig. 6d, e). Interestingly, perilipin 1 levels in the fed state were reduced, which might be a compensatory mechanism to facilitate access of lipases to the lipid droplet TG moiety. These results suggest that $\beta$-adrenergic signalling is not impaired in Stat $5^{\text {Adipoq }}$ mice and indicate that inefficient lipid mobilisation is probably due to reduced levels of ATGL and CGI-58.

STAT5 controls Pnpla2 expression in WAT We next performed a series of experiments to investigate the mechanisms by which STAT5 controls ATGL and CGI-58 levels. Consistent with our in vivo findings, Pnpla2 and Abhd5 mRNA levels in STAT5-deficient explants were reduced, while Lipe expression was unchanged (Fig. 7a, ESM Fig. 6a, b). After GH stimulation for 2 h, Pnpla 2 mRNA was upregulated in control explants, suggesting 
Fig. 5 Impaired lipid mobilisation during fasting in Stat $5^{\text {Adipoq }}$ mice. (a, b) Loss of (a) total fat and (b) lean mass over fed state (baseline) after fasting $(n \geq 6)$. (c-e) Loss of WAT and liver mass over baseline after fasting ( $n \geq 6$ ). (f) Haematoxylin and eosin staining of WAT at baseline and after fasting (staining of WAT at baseline is depicted again here [as well as in Fig. 1d] for the purpose of comparison). Scale bar, $100 \mu \mathrm{m}$. (g) Plasma NEFA and (h) glycerol levels in $48 \mathrm{~h}$ fasted mice $(n \geq 6)$. (i) Liver triacylglycerol content in $48 \mathrm{~h}$ fasted mice. $(\mathbf{j}-\mathbf{m})$ Blood and plasma metabolite and cytokine levels in $48 \mathrm{~h}$ fasted mice. $(n \geq 6)$. $* p<0.05,{ }^{* *} p<0.01$,

$* * * p<0.001$. White bars, control; black bars, Stat $5^{\text {Adipoq }}$
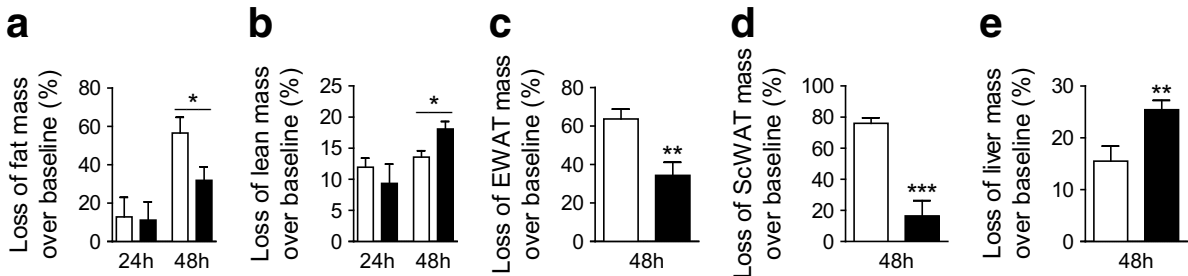

f
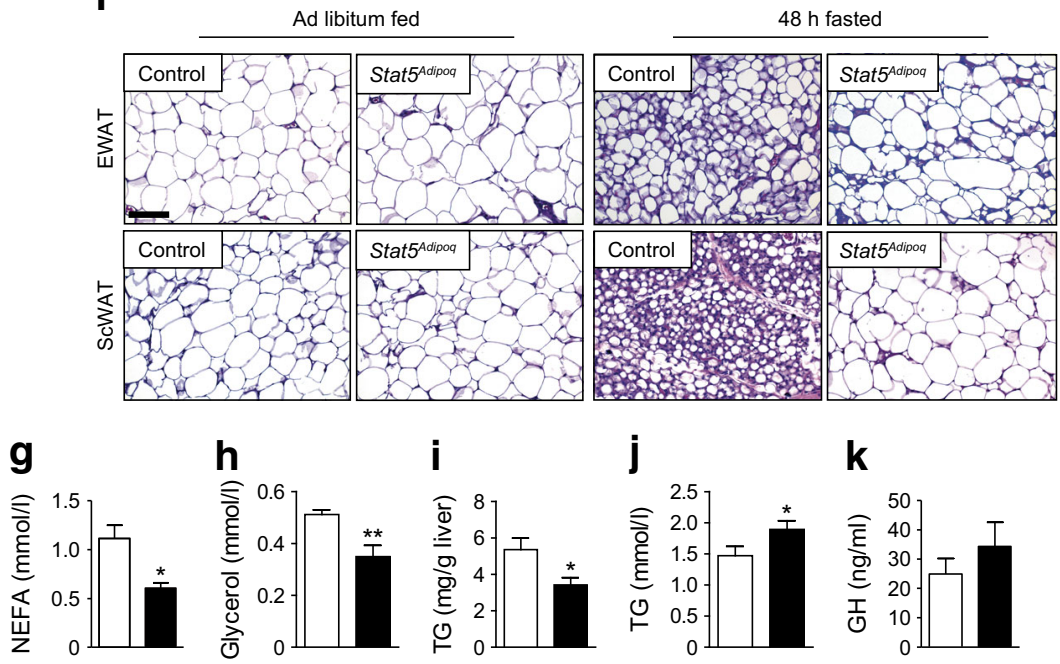

\section{i}
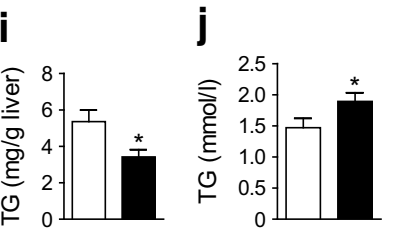

k

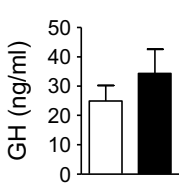

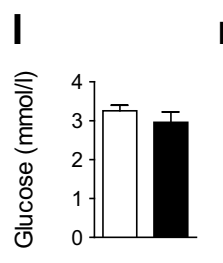

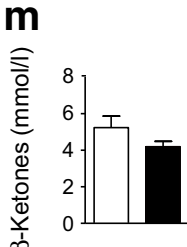

transcriptional activation of the Pnpla2 gene. Consistent with this observation, GH stimulation of 3T3-L1 adipocytes significantly upregulated Pnpla2 and restored insulin-mediated downregulation of Pnpla2 to basal levels ([28], Fig. 7b, ESM Fig. 6c). Moreover, this treatment was sufficient to stimulate Abhd5 upregulation in 3T3-L1 adipocytes (Fig. 7b). Consequently, we investigated whether STAT5 transcriptionally regulates Pnpla 2 and $A b h d 5$. We identified a functional Stat5 response element (RE) within the Pnpla2 promoter region ( -1428 to $-1419 \mathrm{bp})$ and the $A b h d 5$ promoter region $(-1389$ to $-1380 \mathrm{bp})$ by electrophoretic mobility shift assay (Fig. 7c, d). To confirm that STAT5 associates with the identified chromatin regions, we performed ChIP in EWAT extracts. Enriched binding of GH-activated STAT5 was observed on the Pnpla 2 promoter and, to a lesser extent, on the Abhd5 promoter (Fig. 7e). To validate the functionality of the identified REs, we performed reporter assays in NIH3T3 cells in which luciferase gene expression was regulated by either a 146 bp fragment of the Pnpla2 promoter (Pnpla2-luc) or a $138 \mathrm{bp}$ fragment of the Abhd5 promoter (Abhd5-luc) containing the respective Stat5 RE. For Abhd5-luc, reporter gene activity was not induced above empty vector levels (ESM
Fig. 6d); however, Pnpla2-luc had significantly elevated luciferase activity, which was further increased by GH stimulation (Fig. 7f). Mutation of the Stat5 RE within the Pnpla2 promoter fragment (Pnpla2-mutS5RE-luc) decreased luciferase gene expression to empty vector levels. In line with this, Pnpla2-luc reporter activity was abolished in Stat5-null mouse embryonic fibroblasts and restored by Stat 5 a co-expression (ESM Fig. 6e). These data provide mechanistic evidence that STAT5 is a transcriptional regulator of the Pnpla2 gene in WAT.

\section{Discussion}

We report a previously unanticipated requirement for STAT5 in maintaining lipid homeostasis in WAT. Basal lipolysis and fasting-induced lipid mobilisation were diminished by STAT5 deficiency, which was associated with decreased ATGL and CGI-58 levels. We provide further evidence that Pnpla2 is transcriptionally regulated by STAT5 in WAT. Despite increased adiposity, a lack of STAT5 in adipocytes promoted 


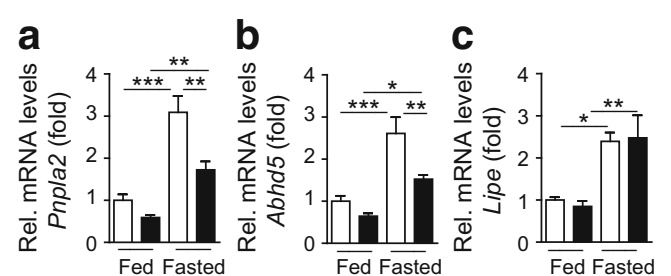

d
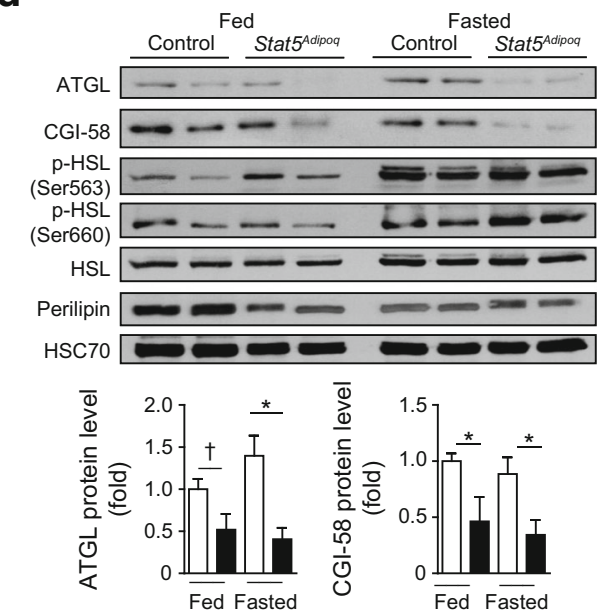

e

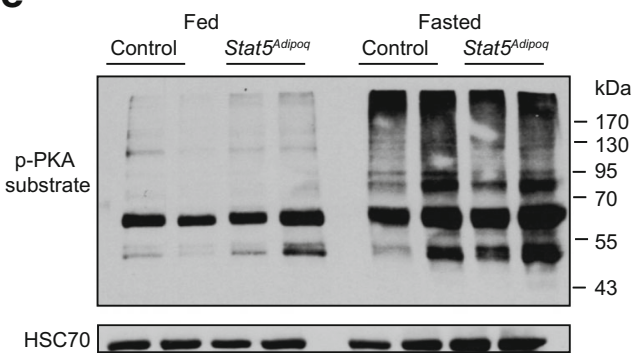

Fig. 6 Impaired lipolysis in Stat $5^{\text {Adipoq }}$ mice is associated with decreased ATGL and CGI-58 levels. (a-c) EWAT mRNA levels for lipolytic genes in fed and $48 \mathrm{~h}$ fasted mice. $\mathrm{C}_{\mathrm{t}}$ values were normalised to Gapdh mRNA levels $(n \geq 5)$. (d, e) Western blot of EWAT lysates shows lipolytic protein activation status and/or total levels $(n \geq 3) . * p<0.05, * * p<0.01$; $* * * p<0.001 ;{ }^{\dagger} p=0.053$. White bars, control; black bars, Stat ${ }^{\text {Adipoq }}$. Rel, relative

improved insulin sensitivity and reduced gluconeogenic capacity in mice.

STAT5 deficiency differentially affected the two main WAT compartments: ScWAT mass was substantially increased despite unaltered adipocyte size, while EWAT mass was unchanged although characterised by adipocyte hypertrophy. Preferential enlargement of ScWAT has been noted upon adipocyte-specific deletion of Jak2 using Adipoq-Cre [21] and in adipose Ghr deleted male mice using aP2-Cre [29], which may reflect different responses to $\mathrm{GH}$ in the respective WAT compartments [30]. Interestingly, adipose Jak2 deletion using aP2-Cre resulted in significant enlargement of all fat depots, although ScWAT showed the greatest increase
[14]. This difference may be attributable to the age, genetic background or Cre-promoter usage. A characteristic feature of both STAT5-deficient WAT compartments was a reduced capacity for basal lipolysis accompanied by decreased mRNA levels for genes involved in FA and TG synthesis (i.e. Acaca, Fasn, Pckl and Dgat2). Interestingly, chronic stimulation of lipolysis was shown to coincide with an upregulation of genes involved in FA and TG synthesis [31], while blockage of lipolysis in chow- and HFD-fed mice resulted in downregulation of these genes [32, 33]. These studies established possible interdependence between lipid mobilisation and synthesis in adipocytes and may explain the reduced expression of lipid storage related genes in STAT5deficient WAT.

Our results indicate that $\beta$-adrenergic responsiveness in Stat $5^{\text {Adipoq }}$ mice is intact, although the lipolysis induction rate was not sufficient to compensate for the defective basal release of lipolytic products upon acute $\beta$-adrenergic stimulation. Similarly, the basal defect was not efficiently compensated for by fasting and thus contributed to aberrant fasting responses. On the molecular level, neither the levels nor activation status of HSL or PKA activity were altered in WAT Stat $5^{\text {Adipoq }}$ mice. This finding supports the observation that impaired lipid mobilisation in Stat ${ }^{\text {Adipoq }}$ mice is not due to defective $\beta$-adrenergic signalling and argues against STAT5dependent regulation of HSL levels in WAT. Adipose STAT5 deficiency resulted in reduced ATGL and CGI-58 levels, thereby impacting the lipolytic machinery at other crucial nodes. In contrast to post-translational regulation of ATGL activity by CGI-58 and G0S2 [3, 34], little is known about the direct transcriptional regulators of Pnpla2: feeding and insulin both repress Pnpla2 expression via the FoxO1 transcription factor, whereas fasting and PPAR $\gamma$ agonists elevate Pnpla2 mRNA levels [2, 3, 28, 35]. Our study extends this knowledge by providing several lines of evidence supporting Pnpla2 regulation by adipose STAT5: (1) Pnpla2 expression is significantly increased by GH only in control WAT explants; (2) STAT5 is enriched at the Pnpla2 promoter in WAT; and (3) a Pnpla2 promoter fragment containing the Stat $5 \mathrm{RE}$ activates a reporter gene. The presence of defects in basal lipolysis in Stat $5^{\text {Adipoq }}$ mice raises the possibility that STAT5 is implicated in balancing lipid metabolism in WAT. This effect may involve pulsatile GH secretion and/or its counter-regulatory effects on insulin function [18] to maintain baseline levels of ATGL and lipid turnover. In support of this notion, GH treatment of 3T3-L1 adipocytes restored insulin-mediated downregulation of Pnpla2 to basal levels. At this point, we cannot exclude the possibility that additional mechanisms contribute, directly or indirectly, to impaired lipolysis in Stat $5^{\text {Adipoq }}$ mice. Given that ATGL activity requires CGI-58 [3], it is conceivable that defective lipolysis results from the simultaneous decrease in the levels of both proteins. However, as we could not 

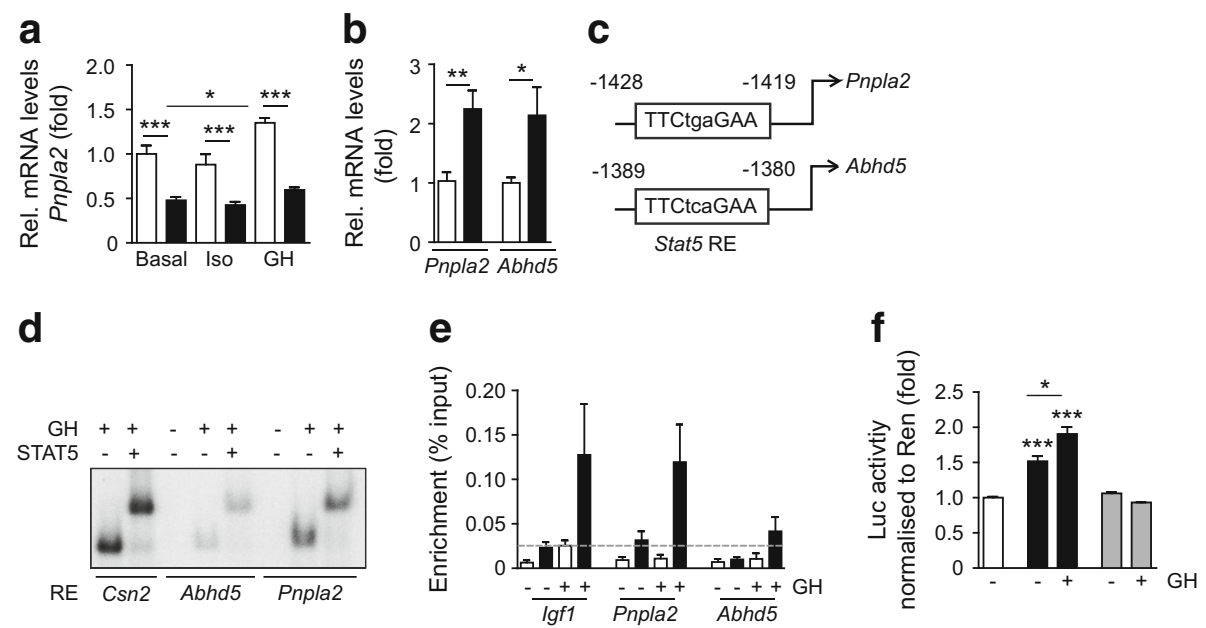

Fig. 7 STAT5 controls Pnpla2 expression in WAT. (a) Pnpla2 mRNA levels in EWAT explants. $\mathrm{C}_{\mathrm{t}}$ values were normalised to Gapdh mRNA levels $(n \geq 6)$. White bars, control; Black bars, Stat $5^{\text {Adipoq }}$. (b) Pnpla2 and $A b h d 5$ mRNA levels in 3T3-L1 adipocytes. $\mathrm{C}_{\mathrm{t}}$ values were normalised to $36 B 4$ mRNA levels $(n=8)$. White bars, PBS; black bars, GH. (c) Putative Stat 5 REs in the promoter regions of Pnpla 2 and Abhd5. Numbers indicate the distance from translation start site. (d) Electrophoretic mobility shift assay. Csn 2 (encoding $\beta$-Casein) RE served as a positive control. (e)

show that the $A b h d 5$ promoter fragment has transactivation potential in reporter assays, further studies are needed to precisely define the underlying molecular mechanism.

STAT5 deficiency in WAT improved variables of glucose metabolism. Adipose tissue has a central role in glucose homeostasis and the lipolytic capacity of WAT negatively correlates with insulin sensitivity $[1,2,32,33]$. Consistent with this, Stat $5^{\text {Adipoq }}$ mice displayed reduced insulin resistance scores and improved insulin sensitivity in ITTs. These results contrast with those of adipocyte Jak2 deletion, which does not alter glucose metabolism in young mice but leads to insulin resistance accompanied by increased circulating NEFA in aged animals [14]. Despite the ability of Stat $5^{\text {Adipoq }}$ mice to maintain euglycaemia during fasting periods, their gluconeogenic capacity in tolerance tests was reduced (i.e. post-hypoglycaemic recovery, gluconeogenesis from pyruvate). It is worth considering that glucose production can be limited by WAT-derived substrate fluxes as glycerol is a direct gluconeogenic substrate, while NEFA oxidation indirectly activates gluconeogenesis via allosteric effectors [36-38]. Hence, diminished NEFA and glycerol availability as well as the depletion of liver glycogen may contribute to the reduced glucose production of Stat $5^{\text {Adipoq }}$ mice.

Collectively, our results identify STAT5 as a pivotal regulator of basal lipolysis that is needed for WAT to respond efficiently to altered energy demands. Moreover, our data indicate that glucose metabolism can be improved by interfering with adipose STAT5. Thus, our study not only extends the current understanding of WAT physiology but also provides a rationale for future studies into the potential of STAT5 manipulation to improve outcomes in metabolic diseases.
ChIP in EWAT. Binding of STAT5 to the $I g f 1$ promoter served as a positive control. The horizontal line indicates the threshold for non-specific binding $(n \geq 5)$. White bars, IgG; black bars, STAT5. (f) Luciferase reporter assay in NIH3T3 cells transfected with the respective reporter construct $(n \geq 5)$. $* p<0.05, * * p<0.01, * * * p<0.001$. White bars, empty vector; black bars, Pnpla2-Luc; grey bars, Pnpla2-mutS5Re-luc. Luc, luciferase; Rel, relative; Ren, Renilla

Acknowledgements We thank S. Zahma (Institute of Animal Breeding and Genetics, University of Veterinary Medicine, Vienna, Austria) for her excellent technical support.

Data availability The data that support the findings of this study are available from the corresponding author upon reasonable request.

Funding This study was supported by grants SFB F28 (F2807-B20) and SFB F47 (SFB F4707-B20) from the Austrian Science Fund and a private research donation from Liechtenstein awarded to RM.

Duality of interest The authors declare that there is no duality of interest associated with this manuscript.

Contribution statement KMM conceptually designed the study. KMM and DK designed the experiments, with contributions from GH and MSchw; KMM and RM supervised the study; DK performed most of the experiments, with contributions from KMM, LK, MSchl, MT, PB and UF; DK performed most of the data analyses, with contributions from LK, MSchl, MT, PB and UF; $\mathrm{DK}, \mathrm{KMM}$ and RM interpreted the data; DK and KMM wrote the manuscript; LK, MSchl, MT, PB and UF edited the manuscript; $\mathrm{GH}$ and MSchw provided intellectual input; and GH, MSchw and RM critically revised the manuscript. All authors approved the final version of the manuscript. RM is responsible for the integrity of the work as a whole.

Open Access This article is distributed under the terms of the Creative Commons Attribution 4.0 International License (http:// creativecommons.org/licenses/by/4.0/), which permits unrestricted use, distribution, and reproduction in any medium, provided you give appropriate credit to the original author(s) and the source, provide a link to the Creative Commons license, and indicate if changes were made. 


\section{References}

1. Rosen ED, Spiegelman BM (2014) What we talk about when we talk about fat. Cell 156:20-44

2. Zechner R, Zimmermann R, Eichmann TO et al (2012) Fat signals - lipases and lipolysis in lipid metabolism and signaling. Cell Metab 15:279-291

3. Lass A, Zimmermann R, Oberer M, Zechner R (2011) Lipolysis - a highly regulated multi-enzyme complex mediates the catabolism of cellular fat stores. Prog Lipid Res 50:14-27

4. Cao H (2014) Adipocytokines in obesity and metabolic disease. J Endocrinol 220:T47-T59

5. Peckett AJ, Wright DC, Riddell MC (2011) The effects of glucocorticoids on adipose tissue lipid metabolism. Metabolism 60: $1500-1510$

6. Desvergne B, Michalik L, Wahli W (2006) Transcriptional regulation of metabolism. Physiol Rev 86:465-514

7. Rohm M, Sommerfeld A, Strzoda D et al (2013) Transcriptional cofactor TBLR1 controls lipid mobilization in white adipose tissue. Cell Metab 17:575-585

8. Eguchi J, Wang X, Yu S et al (2011) Transcriptional control of adipose lipid handling by IRF4. Cell Metab 13:249-259

9. Cernkovich ER, Deng J, Bond MC, Combs TP, Harp JB (2008) Adipose-specific disruption of signal transducer and activator of transcription 3 increases body weight and adiposity. Endocrinology 149:1581-1590

10. Derecka M, Gornicka A, Koralov SB et al (2012) Tyk2 and Stat3 regulate brown adipose tissue differentiation and obesity. Cell Metab 16:814-824

11. Tsoli M, Schweiger M, Vanniasinghe AS et al (2014) Depletion of white adipose tissue in cancer cachexia syndrome is associated with inflammatory signaling and disrupted circadian regulation. PLoS One 9:e92966

12. Moisan A, Lee YK, Zhang JD et al (2015) White-to-brown metabolic conversion of human adipocytes by JAK inhibition. Nat Cell Biol 17:57-67

13. Richard AJ, Stephens JM (2014) The role of JAK-STAT signaling in adipose tissue function. Biochim Biophys Acta 1842:431-439

14. Shi SY, Luk CT, Brunt JJ et al (2014) Adipocyte-specific deficiency of Janus kinase (JAK) 2 in mice impairs lipolysis and increases body weight, and leads to insulin resistance with ageing. Diabetologia 57:1016-1026

15. Jung HS, Lee YJ, Kim YH, Paik S, Kim JW, Lee JW (2012) Peroxisome proliferator-activated receptor gamma/signal transducers and activators of transcription 5A pathway plays a key factor in adipogenesis of human bone marrow-derived stromal cells and 3T3-L1 preadipocytes. Stem Cells Dev 21:465-475

16. Kawai M, Namba N, Mushiake S et al (2007) Growth hormone stimulates adipogenesis of 3T3-L1 cells through activation of the Stat5A/5B-PPAR $\gamma$ pathway. J Mol Endocrinol 38:19-34

17. Moller L, Norrelund H, Jessen $\mathrm{N}$ et al (2009) Impact of growth hormone receptor blockade on substrate metabolism during fasting in healthy subjects. J Clin Endocrinol Metab 94:4524-4532

18. Moller N, Jorgensen JO (2009) Effects of growth hormone on glucose, lipid, and protein metabolism in human subjects. Endocr Rev 30:152-177

19. Berryman DE, Glad CA, List EO, Johannsson G (2013) The GH/ IGF-1 axis in obesity: pathophysiology and therapeutic considerations. Nat Rev Endocrinol 9:346-356

20. Chaves VE, Junior FM, Bertolini GL (2013) The metabolic effects of growth hormone in adipose tissue. Endocrine 44:293-302
21. Nordstrom SM, Tran JL, Sos BC, Wagner KU, Weiss EJ (2013) Disruption of JAK2 in adipocytes impairs lipolysis and improves fatty liver in mice with elevated GH. Mol Endocrinol 27:13331342

22. Berry R, Rodeheffer MS (2013) Characterization of the adipocyte cellular lineage in vivo. Nat Cell Biol 15:302-308

23. Cui Y, Riedlinger G, Miyoshi K et al (2004) Inactivation of Stat5 in mouse mammary epithelium during pregnancy reveals distinct functions in cell proliferation, survival, and differentiation. Mol Cell Biol 24:8037-8047

24. Schlederer M, Mueller KM, Haybaeck J et al (2014) Reliable quantification of protein expression and cellular localization in histological sections. PLoS One 9:e100822

25. Engblom D, Kornfeld JW, Schwake L et al (2007) Direct glucocorticoid receptor-Stat5 interaction in hepatocytes controls body size and maturation-related gene expression. Genes Dev 21:1157-1162

26. Schweiger M, Eichmann TO, Taschler U, Zimmermann R, Zechner R, Lass A (2014) Measurement of lipolysis. Methods Enzymol 538: 171-193

27. Grabner B, Schramek D, Mueller KM et al (2015) Disruption of STAT3 signalling promotes KRAS-induced lung tumorigenesis. Nat Commun 6:6285

28. Kim JY, Tillison K, Lee JH, Rearick DA, Smas CM (2006) The adipose tissue triglyceride lipase ATGL/PNPLA2 is downregulated by insulin and TNF- $\alpha$ in 3T3-L1 adipocytes and is a target for transactivation by PPAR $\gamma$. Am J Phys Endocrinol Metab 291: E115-E127

29. List EO, Berryman DE, Funk K et al (2013) The role of GH in adipose tissue: lessons from adipose-specific GH receptor genedisrupted mice. Mol Endocrinol 27:524-535

30. Berryman DE, List EO, Sackmann-Sala L, Lubbers E, Munn R, Kopchick JJ (2011) Growth hormone and adipose tissue: beyond the adipocyte. Growth Horm IGF Res 21:113-123

31. Mottillo EP, Balasubramanian P, Lee YH, Weng C, Kershaw EE, Granneman JG (2014) Coupling of lipolysis and de novo lipogenesis in brown, beige, and white adipose tissues during chronic $\beta 3$ adrenergic receptor activation. J Lipid Res 55:2276-2286

32. Li YQ, Shrestha YB, Chen M, Chanturiya T, Gavrilova O, Weinstein LS (2016) Gsalpha deficiency in adipose tissue improves glucose metabolism and insulin sensitivity without an effect on body weight. Proc Natl Acad Sci U S A 113:446-451

33. Schreiber R, Hofer P, Taschler U et al (2015) Hypophagia and metabolic adaptations in mice with defective ATGL-mediated lipolysis cause resistance to HFD-induced obesity. Proc Natl Acad Sci U S A 112:13850-13855

34. Yang $\mathrm{X}, \mathrm{Lu} \mathrm{X}$, Lombes $\mathrm{M}$ et al (2010) The $\mathrm{G}_{0} / \mathrm{G}_{1}$ switch gene 2 regulates adipose lipolysis through association with adipose triglyceride lipase. Cell Metab 11:194-205

35. Chakrabarti P, Kandror KV (2009) FoxO1 controls insulindependent adipose triglyceride lipase (ATGL) expression and lipolysis in adipocytes. J Biol Chem 284:13296-13300

36. Samuel VT, Shulman GI (2016) The pathogenesis of insulin resistance: integrating signaling pathways and substrate flux. J Clin Invest 126:12-22

37. Rebrin K, Steil GM, Getty L, Bergman RN (1995) Free fatty acid as a link in the regulation of hepatic glucose output by peripheral insulin. Diabetes 44:1038-1045

38. Perry RJ, Zhang XM, Zhang D et al (2014) Leptin reverses diabetes by suppression of the hypothalamic-pituitary-adrenal axis. Nat Med 20:759-763 